\title{
ASPECTOS HIDROGEOQUÍMICOS E CLASSES DE ÁGUA DO AQUÍFERO CÁRSTICO JANDAÍRA PARA IRRIGAÇÃO, BARAÚNA, RN
}

\section{HYDROGEOCHEMICAL ASPECTS AND WATER CLASSES OF THE KARST JANDAÍRA AQUIFER FOR IRRIGATION, BARAÚNA, RN}

\author{
José Geraldo de Melo ${ }^{1}$, Adriana Barroca de Medeiros ${ }^{1}$, Mickaelon Belchior Vasconcelos ${ }^{2}$ e \\ Vera Lúcia Lopes de Castro ${ }^{3}$
}

\begin{abstract}
RESUMO
A região de Baraúna está situada a Oeste da Bacia Potiguar no domínio semi-árido do Estado do RN. Geologicamente, a região é constituída por rochas carbonáticas (calcários e dolomitos) da Formação Jandaíra de idade atribuída ao Cretáceo Superior. Esta unidade geológica constitui o aquífero cárstico Jandaíra, o qual é a principal fonte de suprimento hídrico da população e de irrigação. O aqüífero Jandaíra, entretanto, desenvolve níveis de carstificação que variam em diferentes setores, influenciando, por conseguinte nas potencialidades e qualidade das águas subterrâneas. Neste artigo são apresentados aspectos relativos ao uso e de salinização das águas subterrâneas, com ênfase as classes de água para irrigação. Foram utilizados dados de estudos geofísicos (eletroresistividade), de cadastro de poços, de estudos topográficos e resultados de análises químicas. Verificou-se que nos setores ao Norte e Oeste de Baraúna, onde as rochas desenvolvem maiores níveis de cárste, as águas são de baixa a média salinidade, do tipo $\mathrm{Ca}-\mathrm{HCO}_{3}$, de um modo geral adequadas para o uso humano e desenvolvimento agrícola (classes $C_{2} S_{1} T_{1}$ e $C_{2} S_{1} T_{2}$ para irrigação); No setor ao Sul de Baraúna, onde as carstes são menos expressivas e ocorrem freqüentes intercalações de folhelho nos calcários, as águas são de elevada salinidade, do tipo $\mathrm{Ca}-\mathrm{Mg}-\mathrm{Cl}$, com restrições de qualidade para uso humano e irrigação (Classe $\mathrm{C}_{3} \mathrm{~S}_{1} \mathrm{~T}_{3}$ para irrigação).
\end{abstract}

Palavras-chave: Bacia Potiguar, Formação Jandaíra, carste, irrigação

\begin{abstract}
The Baraúna region is situated on the West part of the Potiguar Basin, in the semi-arid domain of the RN State. Its geology comprehends mainly carbonate rocks (calcareous and dolomites) of the Jandaíra Formation whose age is attributed to the Superior Cretaceous. This geological unit constitutes the karst Jandaíra Aquifer, which is the main water supply source to human use and irrigation. The Jandaíra Aquifer develops different levels of karst in the different sectors of the area, affecting the groundwater potentialities and its quality. In this article aspects in relation to the groundwater use and salinization are presented, highlighting the groundwater use to irrigation. It was developed using data obtained from geophysical studies, wells cadastre, topographic studies and the interpretation of the hydrochemistry data. In the Northern and Western sectors of Baraúna the karsts are more expressive. In this case the water is $\mathrm{Ca}-\mathrm{HCO}_{3}$ type water, with low salinity and in most of the cases it is good to human use and irrigation (classes $\mathrm{C}_{2} \mathrm{~S}_{1} \mathrm{~T}_{1}$ e C2S1T2 to irrigation); To the Southern part of Baraúna, where the karsts are less expressive, the groundwater salinity is high, $\mathrm{Ca}-\mathrm{Mg}-\mathrm{Cl}$ type water and the quality suffers a certain restriction to human use and irrigation (Class $\mathrm{C}_{3} \mathrm{~S}_{1} \mathrm{~T}_{3}$ ).
\end{abstract}

Keywords: Potiguar Basin, Jandaíra Formation, karst, irrigation

\section{INTRODUÇÃOO}

A área de estudo está situada na Região de Baraúna no extremo Noroeste do Estado do Rio Grande do Norte, cujo limite Oeste se faz com Estado do Ceará, cobrindo uma superfície de aproximadamente $400 \mathrm{~km}^{2}$ (Figura 1). Trata-se de um domínio semi-árido com precipitações pluviométricas médias de $630 \mathrm{~mm}$ anuais e déficit hídrico que alcança $857 \mathrm{~mm}$ anuais (MEDEIROS et al., 2005).

Geologicamente, está situada no Extremo Oeste da Bacia Sedimentar Potiguar, na denominada Plataforma de Aracati, onde ocorrem as rochas carbonáticas da Formação Jandaíra, que corresponde à unidade mais recente da seqüência Cretácea que integra a Bacia Potiguar (Cretáceo Superior). A Formação Jandaíra é constituída por calcários e dolomitos de coloração cinza, desenvolvendo sistemas de fratura e carstes. É comum a presença de intercalações de folhelhos e argilitos nos calcários, conforme tem sido constatado nos perfis dos poços e nos resultados dos estudos geofísicos (FEITOSA, 1994).

As rochas carbonáticas estão sobrepostas a arenitos e argilitos da Formação Açu, do Cretáceo Inferior.

\footnotetext{
${ }^{1}$ Grupo de Pesquisas em Águas Subterrâneas-GPAS, Departamento de Geologia da UFRN ( jgmelo@ ufrnet.br)

${ }^{2}$ Doutorando do Programa de Pós-Graduação em Geociências da UFPE( mickvasconcelos@yahoo.com.br)

${ }^{3}$ SERHID - Secretaria de Recursos Hídricos do Rio Grande do Norte (vcastro.nat@terra.com.br)
} 
Os calcários da Formação Jandaíra na região de Baraúna tem se revelado em geral como um bom condutor hidráulico, decorrente do elevado nível de carstificação, o que lhes atribui o caráter de um aquífero com elevado potencial e água de qualidade adequada ao desenvolvimento da fruticultura irrigada (FEITOSA, 1994). Isto, por conseguinte, tem atraído empresários do setor que vêm implantando em ritmo acelerado perímetros irrigados para a produção de melão, abacaxi e outros, contribuindo substancialmente com a agricultura irrigada e desenvolvimento da região.

Tem-se verificado, nos últimos dez anos, que o número de poços perfurados captando águas do aquífero Jandaíra tem crescido a uma taxa muito elevada, como também tem aumentado as vazões de explotação dessas unidades, com poços produzindo até $200 \mathrm{~m}^{3} / \mathrm{h}$. Paralelamente, tem-se constatado um progressivo rebaixamento do nível da água dos poços na região de Baraúna, culminando no esgotamento/exaustão de diversos poços já existentes e em poços secos resultantes de novas perfurações (DINIZ FILHO, 2003).

Outro fato a ser destacado é quanto a utilização do cloreto de potássio $(\mathrm{KCl})$ como fertilizante na agricultura da Chapada do Apodi, no qual o íon potássio é facilmente assimilado pelo sistema solo-planta, enquanto que o íon cloreto, que é conservativo, é carreado para o aquífero, tornando-se fonte de salinização das águas (FERNANDES et al. 2005).

No presente artigo são discutidos os diferentes contextos hidrogeológicos, hidroquímicos e de salinização das águas, associando essas mudanças às características litológicas e estruturais do terreno, aos rebaixamentos acentuados dos níveis de água, a possível superexplotação do aqüífero e, as atividades antrópicas em geral (agricultura irrigada e desenvolvimento urbano), além de que é feita uma avaliação apurada da qualidade das águas para irrigação.

\section{MÉTODOS}

Os estudos foram desenvolvidos tomando por base o tratamento de dados do cadastro de 602 poços, o nivelamento topográfico de 304 poços (com GPS geodésico) e a utilização dos resultados dos estudos geofísicos pelo método de eletroresistividade (FEITOSA, 1994). Essas atividades foram desenvolvidas pela Secretaria de Recursos Hídricos do Rio Grande do Norte (SERHID/RN) através da Fundação de Apoio ao Desenvolvimento da UFPE (FADE), as quais permitiram a concepção da estrutura hidrogeológica do terreno e a elaboração do mapa de fluxo das águas subterrâneas.

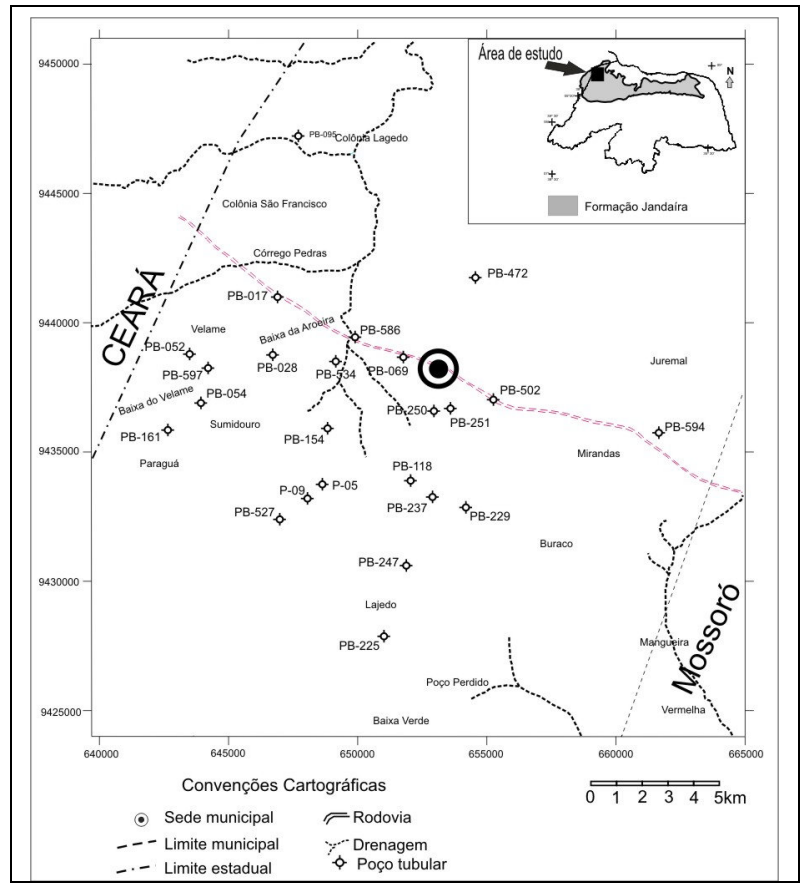

Figura 1 - Situação da área de estudo e distribuição dos poços da rede de monitoramento.

Figure 1 - Study area and wells distribution of the monitoring network.

$\mathrm{O}$ reconhecimento da qualidade das águas foi inicialmente efetuado através das medições da condutividade elétrica das águas com determinações in situ utilizando condutivímetro portátil. Em seguida, o comportamento hidrogeoquímico e a qualidade das águas foram avaliados tomando por base a interpretação dos resultados das análises de 24 amostras de água de poço obtidas em março de 2004. 


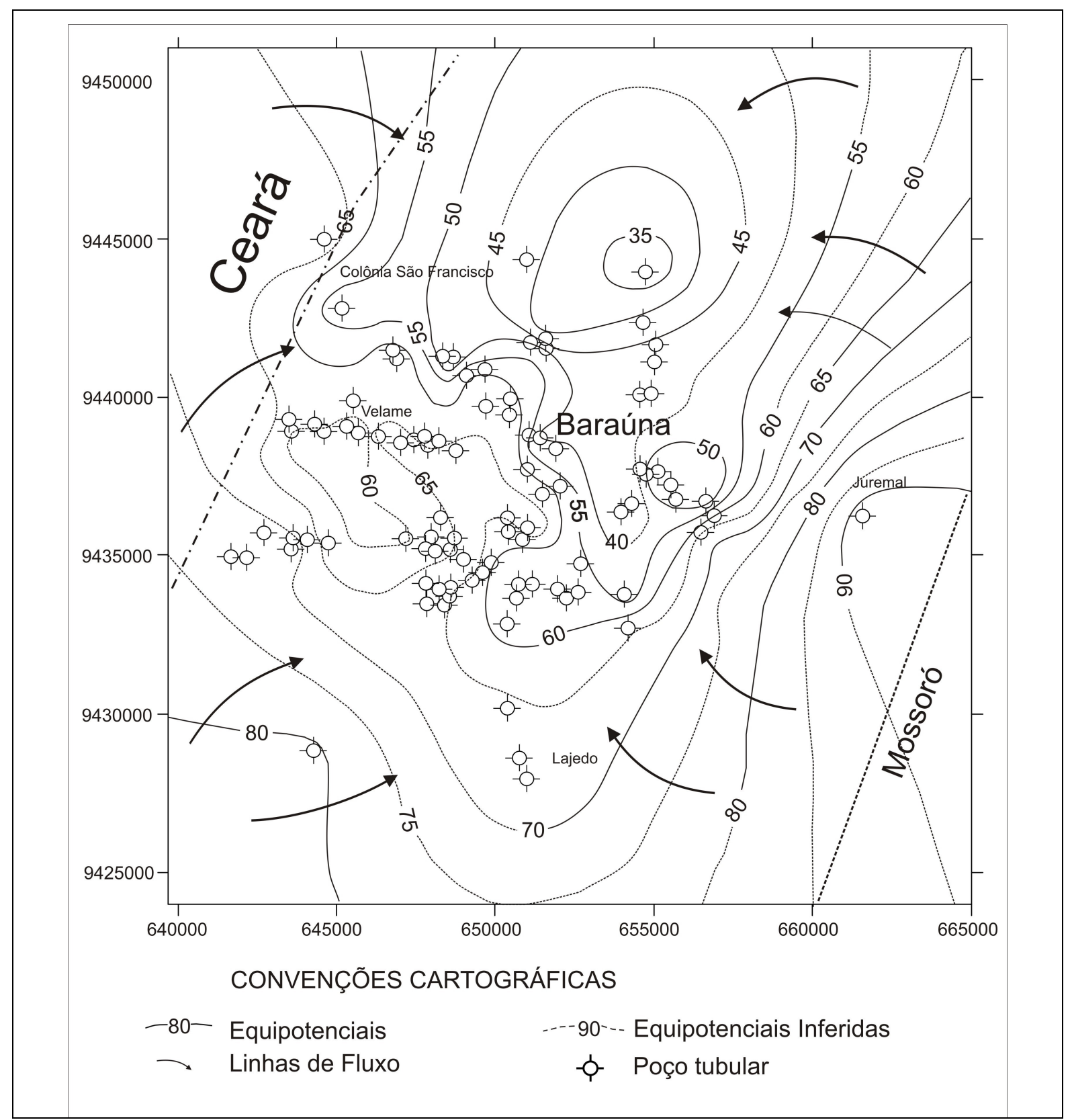

Figura 2 - Superfície potenciométrica do aqüífero Jandaíra na região de Baraúna, RN.

Figure 2 - Potentiometric surface of Jandaíra Aquifer in the Baraúna Region, RN

Os resultados das análises foram submetidos a um balanço iônico, o qual indicou erro, em geral, inferior a $5 \%$, mostrando assim a boa qualidade dos dados. Os parâmetros determinados incluem os cátions e ânions principais $\mathrm{Na}^{+}, \mathrm{K}^{+}, \mathrm{Ca}^{2+}$, $\mathrm{Mg}^{2+}, \mathrm{Cl}^{-}, \mathrm{SO}_{4}{ }^{2-}$ e $\mathrm{HCO}_{3}^{-}$, além dos componentes da série nitrogenada $\left(\mathrm{NH}_{3}, \mathrm{NO}_{2}^{-}\right.$e $\left.\mathrm{NO}_{3}^{-}\right)$e do $\mathrm{CO}_{3}^{2-}, \mathrm{Fe}$, dureza, alcalinidade, condutividade elétrica e $\mathrm{pH}$.

\section{PARÂMETROS HIDRÁULICOS E FLUXO SUBTERRÂNEO}

Os poços perfurados na área apresentam profundidades que variam de 60 a $120 \mathrm{~m}$, com a ocorrência de poços desde secos a poços produzindo até $200 \mathrm{~m}^{3} / \mathrm{h}$. Os níveis estáticos dos poços variam de 15 a $50 \mathrm{~m}$ e são produzidos rebaixamentos que geram níveis dinâmicos de 16 a $70 \mathrm{~m}$.

A superfície potenciométrica do aqüífero se acha bastante afetada, com depressões provavelmente causadas pela superexplotação das águas subterrâneas na parte central da área na região sul e sudoeste de Baraúna, denotando-se, entretanto uma tendência do fluxo subterrâneo regional na direção de Sudoeste para Nordeste (Figura 2).

\section{ANÁLISE HIDROGEOQUÍMICA E QUALIDADE DAS ÁGUAS}

As considerações e discussões apresentadas a seguir dizem respeito aos resultados das análises 
químicas de uma campanha de coleta de amostras de água realizada nos meses de março e abril de 2004 (Tabela 1).

\section{RESULTADOS DAS ANÁLISES QUÍMICAS}

A condutividade elétrica das águas variou de $845 \mu \mathrm{S} / \mathrm{cm}$ (setor Noroeste) a $2060 \mu \mathrm{S} / \mathrm{cm}$ (parte central da área). Os sólidos totais dissolvidos, de $508,0 \mathrm{mg} / \mathrm{L}$ a $1334,0 \mathrm{mg} / \mathrm{L}$ e a concentração de cloreto, de um mínimo de $101,0 \mathrm{mg} / \mathrm{L}$ a um máximo 484,0 $\mathrm{mg} / \mathrm{L}$. De um modo geral a salinidade das águas é mais elevada nos setores Sudoeste e Central da área (Figura 3), que corresponde aos domínios onde a Formação Jandaíra apresenta maiores intercalações de folhelho e calcários mais puros (Figura 4). $\mathrm{O} \mathrm{pH}$ das águas tende a básico, com valores que variam de 7,1 a 7,8 . Os teores de nitrato situam-se entre 7,0 a $22,0 \mathrm{mg} / \mathrm{L}$ de $\mathrm{N}$, estando a maioria dos poços com concentrações superiores a $10 \mathrm{mg} / \mathrm{L}$ de N (Tabela 1), que é o limite estabelecido pela Ministério da Saúde (BRASIL, 2004) para o consumo humano, o que se atribui a intensa prática da agricultura irrigada com o uso de fertilizantes e defensivos agrícolas.

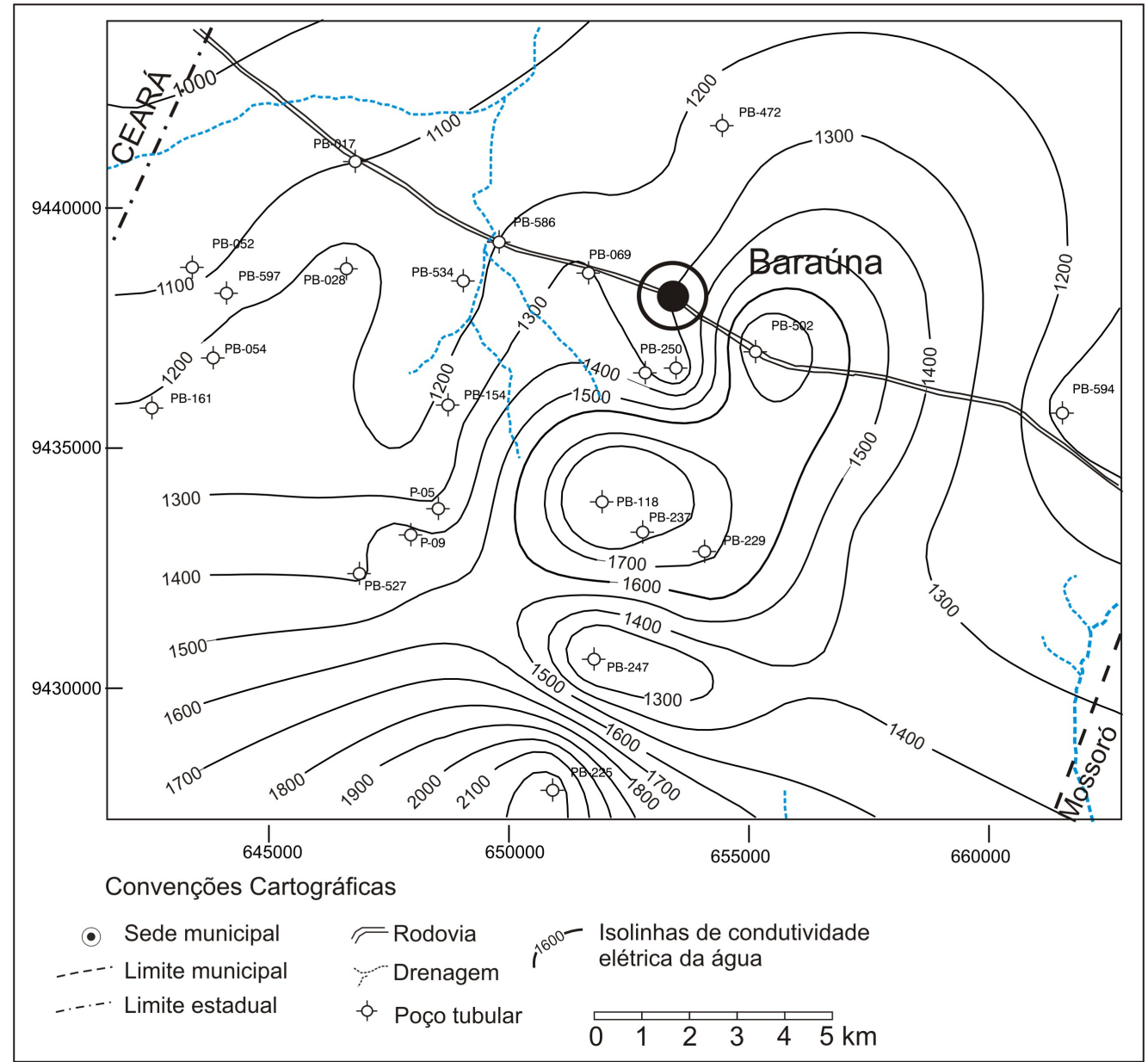

Figura 3 - Condutividade elétrica das águas subterrâneas

Figure 3 - Groundwater electric conductivity 
Tabela 1 - Resultados das análises químicas das águas

Table 1 - Groundwater chemical analysis results

\begin{tabular}{|c|c|c|c|c|c|c|c|c|c|c|c|}
\hline Poço & $\mathrm{pH}$ & $\begin{array}{c}\mathrm{CE} \\
(\mu \mathrm{S} / \mathrm{cm})\end{array}$ & $\begin{array}{r}\mathrm{NH}_{3} \\
(\mathrm{~m}\end{array}$ & $\begin{array}{l}\mathrm{NO}_{3} \\
-\mathrm{N})\end{array}$ & $\mathrm{Ca}^{2+}$ & $\mathrm{Mg}^{2+}$ & $\mathrm{Na}^{+}$ & $\begin{array}{c}\mathrm{K}^{+} \\
(\mathrm{mg} / \mathrm{L})\end{array}$ & $\mathrm{HCO}_{3}^{-}$ & $\mathrm{SO}_{4}^{2-}$ & $\mathrm{Cl}^{-}$ \\
\hline P-05 & 7,3 & 1273 & 0,5 & 12,4 & 112,3 & 38,62 & 16,1 & 3,3 & 284,4 & 35,2 & 151,4 \\
\hline P-09 & 7,2 & 1504 & 0,3 & 16,2 & 144,1 & 44,3 & 16,1 & 3,8 & 323,9 & 48,3 & 185,8 \\
\hline PB-017 & 7,4 & 844 & 0,0 & 6,9 & 74,9 & 27,2 & 41,6 & 2,2 & 208,6 & 31,6 & 101,2 \\
\hline PB-028 & 7,1 & 1249 & 0,4 & 9,6 & 138,5 & 34,0 & 10,4 & 2,7 & 323,9 & 29,4 & 144,5 \\
\hline PB-052 & 7,2 & 1064 & 0,5 & 6,9 & 121,7 & 29,5 & 10,4 & 2,2 & 337,4 & 30,0 & 110,1 \\
\hline PB-054 & 7,2 & 1249 & 0,5 & 10,3 & 121,7 & 32,9 & 15,2 & 3,3 & 307,3 & 32,0 & 144,5 \\
\hline PB-069 & 7,3 & 1087 & 0,0 & 11,2 & 106,7 & 34,0 & 54,1 & 3,3 & 263,7 & 37,8 & 150,3 \\
\hline PB-095 & 7,3 & 983 & 0,4 & 11,3 & 97,3 & 20,4 & 14,2 & 2,7 & 211,7 & 36,3 & 103,2 \\
\hline PB-118 & 7,2 & 1921 & 0,0 & 15,7 & 179,7 & 55,6 & 142,8 & 7,2 & 350,9 & 78,7 & 411,7 \\
\hline PB-154 & 7,3 & 1342 & 0,5 & 16,7 & 138,5 & 31 , & 16,1 & 3,3 & 319,7 & 27,6 & 144,5 \\
\hline PB-161 & 7,4 & 1273 & 0,5 & 12,4 & 116,0 & 32,9 & 15,2 & 2,7 & 290,7 & 29,9 & 137,6 \\
\hline PB-225 & 7,6 & 1921 & 0,0 & 15,9 & 187,2 & 59,0 & 150,0 & 3,8 & 360,2 & 64,7 & 379,0 \\
\hline PB-229 & 7,8 & 1921 & 0,2 & 17,9 & 150,2 & 45,4 & 159,0 & 8,3 & 328,0 & 71,3 & 343,1 \\
\hline PB-237 & 7,6 & 1921 & 0,2 & 14,8 & 140,0 & 58,5 & 140,0 & 5,8 & 285,5 & 66,3 & 372,5 \\
\hline PB-247 & 7,6 & 2060 & 0,0 & 22,1 & 149,8 & 69,2 & 164,2 & 8,6 & 195,1 & 86,4 & 483,6 \\
\hline PB-250 & 7,6 & 1388 & 0,8 & 9,0 & 105,2 & 42,5 & 72,7 & 3,0 & 295,8 & 39,7 & 195,5 \\
\hline PB-251 & 7,5 & 1319 & 0,7 & 9,1 & 126,4 & 36,3 & 63,6 & 3,2 & 352,9 & 34,8 & 168,2 \\
\hline PB-472 & 7,5 & 1249 & 0,1 & 15,8 & 106,8 & 27,2 & 68,3 & 3,0 & 255,4 & 35,8 & 149,5 \\
\hline PB-502 & 7,6 & 1504 & 0,0 & 9,8 & 104,8 & 61,3 & 87,5 & 6,3 & 203,4 & 73,7 & 307,1 \\
\hline PB-527 & 7,2 & 1458 & 0,9 & 21,2 & 136,6 & 37,4 & 16,1 & 2,7 & 268,8 & 33,8 & 165,2 \\
\hline PB-534 & 7,7 & 1203 & 0,2 & 14,0 & 125,0 & 30,9 & 53,5 & 3,0 & 316,6 & 30,4 & 140,5 \\
\hline PB-586 & 7,6 & 1111 & 0,0 & 10,7 & 136,6 & 31,0 & 37,5 & 2,7 & 334,3 & 31,1 & 130,7 \\
\hline PB-594 & 7,2 & 1064 & 0,8 & 9,6 & 102,9 & 29,5 & 10,4 & 2,7 & 246,0 & 28,0 & 117,0 \\
\hline PB-597 & 7,4 & 1226 & 0,6 & 9,2 & 104,3 & 30,1 & 59,0 & 2,5 & 275,1 & 31,1 & 145,5 \\
\hline
\end{tabular}




\section{TIPOS DE ÁGUA}

Foram definidos dois grupos de água ou fácies hidroquímicas (Figura 5). O grupo 1 está situado no setor Sudoeste da área e é caracterizado dominantemente por águas do tipo Ca-Mg-Cl. Neste setor é freqüente a presença de intercalações argilosas nos calcários, tendo sido preservada a característica regional de águas cloretadas. O grupo 2, situado no setores Norte e Oeste da área, é formado por águas que tendem a $\mathrm{Ca}-\mathrm{HCO}_{3}$.

Neste caso, os calcários são mais puros e o desenvolvimento de carstificações é mais proeminente, o que resulta numa maior dissolução $\mathrm{CaCO}_{3}$ e maior ocorrência dos íons bicarbonato e cálcio.

A presença de $\mathrm{Ca}^{2+}$ e $\mathrm{Mg}^{2+}$, nos dois casos estão associadas à dissolução de calcitas e dolomitas.

\section{AVALIAÇÃo DA QUALIDADE DA ÁGUA PARA IRRIGAÇÃO}

A prática da irrigação é indispensável nas regiões áridas e semi-áridas em virtude da ocorrência de déficit hídrico para as culturas na época seca. A acumulação de água no período chuvoso alternado com aumento da evaporação na estação seca promove uma variação quantitativa e qualitativa no teor de sais das águas com implicações para uso na irrigação (NUNES FILHO et al., 1991) e esta, pode ser fator de salinização quando não manejada adequadamente (PIZARRO, 1985; HOORN; ALPHEN, 1981). É, portanto, imprescindível a avaliação da qualidade da água como medida preventiva dos processos de salinização gradativos pelo acumulo de sais oriundos de irrigações sucessivas (HOLANDA; AMORIM, 1992).

A qualidade das águas da região de Baraúna foi avaliada sob os três aspectos fundamentais quanto ao uso na irrigação: salinidade (C), sodicidade (S) e toxidade (T) de íons. O efeito da salinidade é de natureza osmótica podendo afetar diretamente o rendimento das culturas. A sodicidade se refere ao efeito relativo do sódio da água de irrigação tendendo a elevar a porcentagem de sódio trocável do solo (PST), com danos nas suas propriedades físico-químicas, provocando problemas de infiltração. A toxidade, diz respeito ao efeito específico de certos íons sobre as plantas, afetando o rendimento, independente do efeito osmótico.

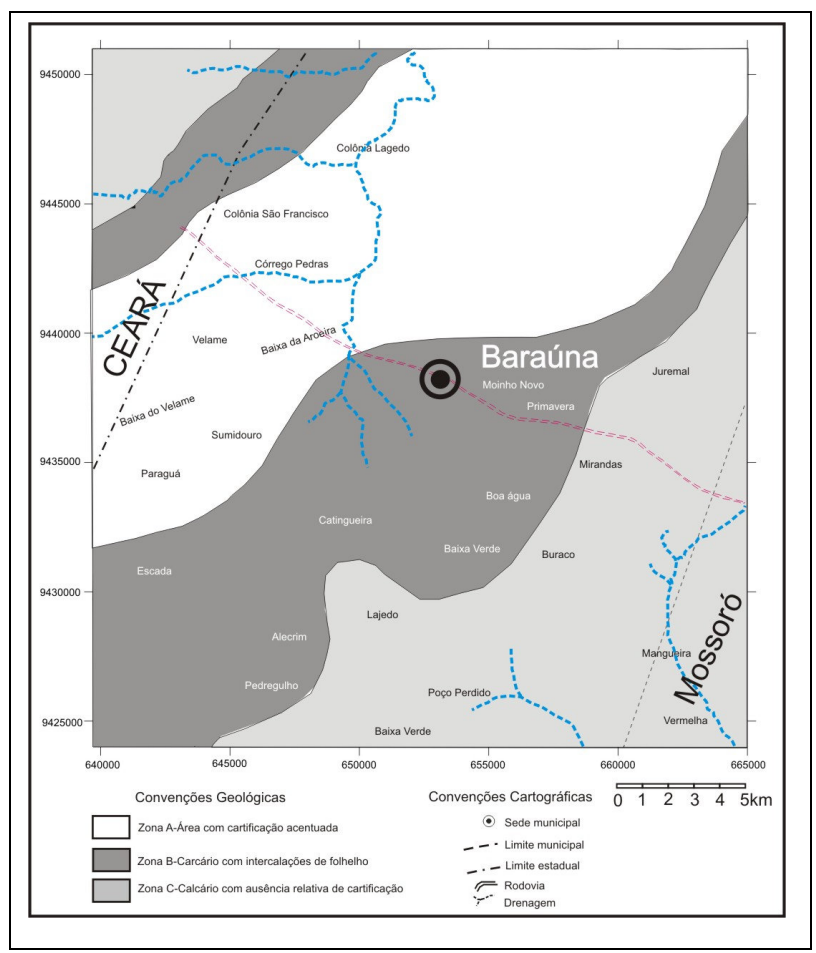

Figura 4 - Fácies litológicas nos calcários da Formação Jandaíra (FEITOSA,1994).

Figure 4 - Litological "facies" in the Jandaíra Aquifer Calcareous

\section{CLASSES DE ÁGUA QUANTO AO RISCO DE SALINIDADE}

O excesso de sais na zona radicular afeta consideravelmente o desenvolvimento das culturas, com diminuição na taxa de respiração e crescimento (HOLANDA; AMORIM, 1992). A excessiva salinidade reduz o desenvolvimento devido ao aumento de energia que precisa ser despendida para absorver água do solo a ao ajustamento bioquímico necessário para sobreviver sob estresse (RHOADES et al., 1992).

A condutividade elétrica é o parâmetro empregado para expressar a concentração de sais solúveis na água tendo em vista a elevada correlação que existe com os sólidos totais dissolvidos. 
Tabela 2: Classificação da água para irrigação quanto ao risco de salinidade.

Table 2: Classification of the water for irrigation in relation to the salinity risks.

\begin{tabular}{|c|c|c|c|c|c|}
\hline \multirow{2}{*}{$\begin{array}{l}\text { Classe de } \\
\text { Salinidade }\end{array}$} & Richards(1954) & $\mathrm{UCCC}^{1}$ & \multirow{2}{*}{$\begin{array}{l}\text { Risco de } \\
\text { Salinidade }\end{array}$} & \multicolumn{2}{|c|}{ Ayers; Westcot (1991) } \\
\hline & Faixas de $\mathrm{Cl}$ & IS.m $\left.\mathrm{m}^{-1}\right)$ & & Faixa de $\mathrm{CE}_{\mathrm{a}}\left(\mathrm{dS} \cdot \mathrm{m}^{-1}\right)$ & Problema de \\
\hline $\mathrm{C}_{1}$ & $<0,25$ & $<0.75$ & Baixo & $<07$ & Nenhum \\
\hline $\mathrm{C}_{2}$ & $0,25-0,75$ & $0,75-1,50$ & Médio & $0,7-3,0$ & moderado \\
\hline $\mathrm{C}_{3}$ & $0,75-2,25$ & $1,50-3,00$ & Alto & $>3,0$ & Severo \\
\hline $\mathrm{C}_{4}$ & $>2,25$ & $>3,00$ & Muito alto & - & - \\
\hline
\end{tabular}

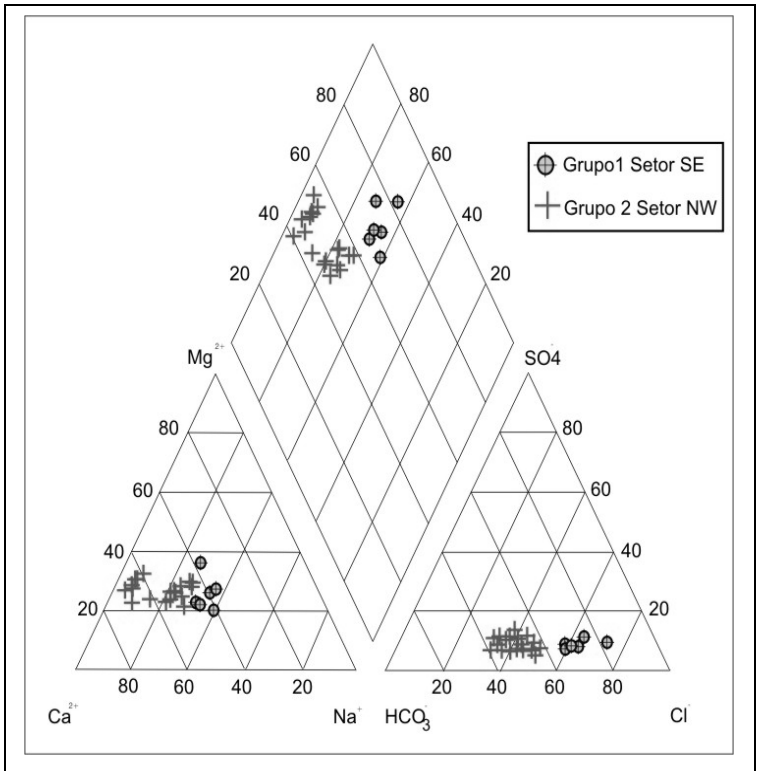

Figura 5 - Diagrama de Piper (Aqüífero Jandaíra na região de Baraúna).

Figure 5 - Piper diagram (Jandaíra Aquifer in the Baraúna Region)

Numa avaliação qualitativa, as águas se dividem em quatro classes de salinidade, à medida que aumenta a concentração de sais e conseqüentemente sua condutividade elétrica, recebendo denominações sucessivas de $\mathrm{C}_{1}, \mathrm{C}_{2}$, $\mathrm{C}_{3}$ e $\mathrm{C}_{4}$, conforme apresentado a seguir:

- $\mathrm{C}_{1}$ - Água de baixa salinidade. Pode ser usada para irrigação da maioria das culturas, em quase todos os tipos de solos, com muito pouca probabilidade de que se desenvolvam problemas de salinidade.

- $\mathrm{C}_{2}$ - Água de média salinidade. Pode ser usada sempre e quando houver uma lixiviação moderada de sais. Em quase todos os casos permitem o cultivo de plantas moderadamente tolerante aos sais, sem necessidade de práticas especiais de controle de salinidade.
- $\mathrm{C}_{3}$ - Água de alta salinidade. Não pode ser usada em solos com drenagem deficiente. Mesmo com drenagem adequada podem ser necessárias práticas especiais de controle da salinidade, devendo, portanto, ser utilizada na irrigação de espécies vegetais de alta tolerância aos sais. Os riscos apresentados por esta classe de água podem ser amenizados quando do emprego do método de irrigação localizada mantendo o solo continuamente úmido.

- $\mathrm{C}_{4}$ - Água de muito alta salinidade. Não é apropriada para irrigação sob condições normais, porém pode ser usada ocasionalmente, em circunstâncias muito especiais. Os solos devem ser permeáveis, a drenagem adequada, devendo ser aplicada água em excesso para se obter uma boa lixiviação de sais e, mesmo assim devem ser explorados com culturas altamente tolerantes aos sais.

Em sua forma mais moderna, a classificação das águas quanto ao risco de salinidade foi estabelecida segundo as proposições da UCCC - University of California Commitee of Consultants (FRENKEL,1984; PIZARRO, 1985; AYERS; WESTCOT, 1991), que estabeleceram limites diferenciados para aqueles propostos por Richards (1954) tabela 2.

A salinidade afeta tanto o crescimento das plantas quanto a produção e qualidade do produto, se manifestando principalmente na redução da população e desenvolvimento das culturas, com sintoma similar ao causado por estresse hídrico (RHOADES et al., 1992). 


\section{CLASSES DE ÁGUA QUANTO AO RISCO DE SODICIDADE}

Está amplamente comprovado que valores altos de percentual de sódio trocável (PST), especialmente sob condições de baixa salinidade, causam a dispersão de partículas com redução na condutividade hidráulica do solo (HOLANDA; AMORIM, 1992). A razão de adsorção de sódio (RAS) na água de irrigação é o parâmetro de melhor correlação com a PST do solo. Richards (1954) foi o primeiro a introduzir esse conceito num sistema de classificação de águas para irrigação que passou a ser usado e conhecido internacionalmente pelo seu próprio nome. Ultimamente, o risco de sodicidade passou a ser avaliado com mais segurança relacionando a RAS corrigida $\left(\mathrm{RAS}^{\circ}\right)$ com a salinidade da água, estimada pela equação 1 :

$$
R A S^{o}=\frac{N a}{\sqrt{\frac{\left(C a^{o}+M g\right)}{2}}}
$$

Sendo:

$\mathrm{Na}^{+}$- Concentração de sódio na água, em $\mathrm{mmol}_{\mathrm{c}} \mathrm{L}^{-1}$; $\mathrm{Ca}^{\mathrm{o}}$ - Concentração de cálcio na água, corrigida pela relação $\mathrm{HCO}_{3}^{-} / \mathrm{Ca}\left(\mathrm{mmol}_{\mathrm{c}} \mathrm{L}^{-1}\right)$ e $\mathrm{CE}_{\mathrm{a}}\left(\mathrm{dS} \cdot \mathrm{m}^{-1}\right)$, de acordo com a tabela 3;

$\mathrm{Mg}^{2+}$ - Concentração de Magnésio na água, em mmol $_{\mathrm{c}} \mathrm{L}^{-1}$.

A $\mathrm{RAS}^{\mathrm{o}}$, conforme procedimento sugerido por Suarez (1981), facilita o entendimento das alterações que ocorrem com o cálcio na água do solo em função da dissolução dos carbonatos e silicatos.

A classificação das águas de irrigação com respeito a RAS se baseia essencialmente no efeito do sódio trocável nas condições físicas do solo causando problemas de infiltração pela redução da permeabilidade. Richards (1954) dividiu as águas em quatro classes tomando por base valores limites da RAS, em função da $\mathrm{CE}_{\mathrm{a}}$, com as seguintes interpretações:

- $\mathrm{S}_{1}\left(\mathrm{RAS}<18,87\right.$ - 4,44.log $\left.\mathrm{CE}_{\mathrm{a}}\right)$ - água de baixa sodicidade ou com baixa concentração de sódio. Podem ser usados para irrigação na maioria dos solos, com pouca probabilidade de se atingir níveis perigosos de sódio trocável.

- $\mathrm{S}_{2}\left(18,87\right.$ - 4,44.log $\mathrm{CE}_{\mathrm{a}}<\mathrm{RAS}<31,31$ 6,66.log $\mathrm{CE}_{\mathrm{a}}$ ) - água de sodicidade média ou com média concentração de sódio. Em solos de textura fina (argilosos) o sódio dessa classe de água representa um perigo considerável de dispersão com redução de permeabilidade. Por outro lado, essas águas podem ser usadas em solos de textura grossa (arenosos) ou em solos orgânicos de boa permeabilidade.

- $\mathrm{S}_{3}\left(31,31\right.$ - 6,66.log $\mathrm{CE}_{\mathrm{a}}<\mathrm{RAS}<43,75$ 8,87.log $\left.\mathrm{CE}_{\mathrm{a}}\right)$ - água de alta sodicidade ou com alta concentração de sódio. Pode produzir níveis tóxicos de sódio trocável na maioria dos solos, necessitando de práticas especiais de manejo: boa drenagem, alta lixiviação e adição de condicionadores químicos ou orgânicos.

- $\mathrm{S}_{4}\left(\mathrm{RAS}>43,75\right.$ - 8,87.log $\mathrm{CE}_{\mathrm{a}}$ ) - água de muito alta sodicidade ou de muito alta concentração de sódio. É uma classe inadequada para irrigação exceto quando sua salinidade é baixa ou média.

A classificação de Richards (1954) é a mais utilizada até hoje, porém a mesma tem sido questionada Pizarro (1985) pelos motivos a seguir:

A suposição de que o cálcio e magnésio têm a mesma seletividade de troca iônica, o que não corresponde a realidade; para uma mesma RAS a adsorção de sódio cresce ao aumentar a relação $\mathrm{Mg} / \mathrm{Ca}$ devido a menor energia de adsorção do magnésio;

Não leva em conta a possibilidade de precipitação de sais, fenômeno que pode aumentar o risco de sodicidade, já que o cálcio é o cátion mais sujeito a reação, precipitando na forma de carbonato e sulfato que são de baixa solubilidade;

Também, a classificação tem um erro conceitual, pois, os sais da solução do solo têm um efeito floculante, oposto ao efeito dispersante do sódio trocável. Dessa forma, para uma mesma RAS o risco de sodicidade será menor quanto maior for a CEa. Assim, as linhas descendentes no diagrama de classificação passam a ser ascendentes, conforme mostrado na figura 5, traçada empiricamente com os valores das classes de salinidade do UCCC, citado por Pizarro (1985) e das classes de sodicidade/infiltração apresentada por Ayers; Westcot (1991).

Das dezesseis classes previstas da combinação da salinidade com a sodicidade na classificação de Richards (1954), seis não existem sob condições naturais (BHUMBLA, 1977).

A recomendação de AYERS; WESTCOT (1991), quanto ao perigo de sódio, restringe-se a 
três classes de sodicidade, obtidas relacionandose a RAS $^{0}$ com a salinidade da água de irrigação (Tabela 3).

CLASSES DE ÁGUA QUANTO AO RISCO DE TOXICIDADE

Os íons cloreto, sódio e boro, quando presentes em concentrações elevadas na água, podem causar danos às culturas reduzindo a produção. A magnitude do dano depende, além da concentração do íon, do tempo de exposição, da sensibilidade das plantas, do uso da água pelas culturas, do tipo de irrigação, entre outros (MAAS, 1985). Em irrigação por aspersão e sob condições de alta evaporação o problema tende a se acentuar. As culturas arbóreas e plantas lenhosas em geral são sensíveis ao sódio e cloreto (MAAS, 1986).

Foram definidas três classes de risco quanto a toxidade das plantas (AYERS; WESTCOT, 1991)designadas de T1, T2 e T3, os quais se acham definidos na tabela 5 .

Tabela 3 - Riscos de problemas de infiltração no solo causados pela sodicidade da água.

Table 3 - Irrigation water classification diagram (RICHARDS, 1954)

\begin{tabular}{cccc}
\hline & \multicolumn{3}{c}{ Classes de sodicidade $^{1}$} \\
\cline { 2 - 4 } RAS $^{\mathrm{N}}$ & $\mathrm{S}_{1}$ & $\mathrm{~S}_{2}$ & $\mathrm{~S}_{3}$ \\
& $\begin{array}{c}\text { Sem } \\
\text { Problemas }\end{array}$ & $\begin{array}{c}\text { Problemas } \\
\text { Crescentes }\end{array}$ & $\begin{array}{c}\text { Problemas } \\
\text { Severos }\end{array}$ \\
\hline$\left(\mathrm{mmol}_{\mathrm{c}} \mathrm{L}^{-1}\right)^{1 / 2}$ & $\mathrm{CE}_{\mathrm{a}}\left(\mathrm{dS} \cdot \mathrm{m}^{-1}\right)$ & \\
$0-3$ & $>0,70$ & $0,70-0,20$ & $<0,20$ \\
$3-6$ & $>1,20$ & $1,20-0,30$ & $<0,30$ \\
$6-12$ & $>1,90$ & $1,90-0,50$ & $<0,50$ \\
$12-20$ & $>2,90$ & $2,90-1,30$ & $<1,30$ \\
$20-40$ & $>5,00$ & $5,00-2,90$ & $<2,90$ \\
\hline
\end{tabular}

Fonte: Adaptado de Ayers; Westcot (1991). ${ }^{1}$ Simbologia (S) não se refere a classificação de Richards (1954); foi inserida para resumir a descrição da classe.

Tabela 4 - Concentração de cálcio $\left(\mathrm{Ca}^{2+}\right)$ contida na água do solo, próximo à superfície, que resultaria da irrigação com água de determinada relação $\mathrm{HCO}_{3} / \mathrm{Ca}$ e $\mathrm{CE}^{1,2,3}$

Table 4 - Calcio $\left(\mathrm{Ca}^{2+}\right)$ concentration in the soil water as a result of water irrigation for the relation $\mathrm{HCO}_{3} / \mathrm{Ca}$ $C E^{1,2,3}$

\begin{tabular}{|c|c|c|c|c|c|c|c|c|c|c|c|c|}
\hline \multirow{2}{*}{$\begin{array}{r}\text { Valor de } \\
\mathrm{HCO}_{3} / \mathrm{Ca} \\
\text { da água } \\
\end{array}$} & \multicolumn{12}{|c|}{ Salinidade da Água Aplicada (CE) - dS.m ${ }^{-1}$} \\
\hline & 0,1 & 0,2 & 0,3 & 0,5 & 0,7 & 1,0 & 1,5 & 2,0 & 3,0 & 4,0 & 6,0 & 8,0 \\
\hline 0,05 & 13,20 & 13,61 & 13,92 & 14,40 & 14,79 & 15,26 & 15,91 & 16,43 & 17,28 & 17,97 & 19,07 & 19,94 \\
\hline 0,10 & 8,31 & 8,57 & 8,77 & 9,07 & 9,31 & 9,62 & 10,02 & 10,35 & 10,89 & 11,32 & 12,01 & 12,56 \\
\hline 0,15 & 6,34 & 6,54 & 6,69 & 6,92 & 7,11 & 7,34 & 7,65 & 7,90 & 8,31 & 8,64 & 9,17 & 9,58 \\
\hline 0,20 & 5,24 & 5,40 & 5,52 & 5,71 & 5,87 & 6,06 & 6,31 & 6,52 & 6,86 & 7,13 & 7,57 & 7,91 \\
\hline 0,25 & 4,51 & 4,65 & 4,76 & 4,92 & 5,06 & 5,22 & 5,44 & 5,62 & 5,91 & 6,15 & 6,52 & 6,82 \\
\hline 0,30 & 4,00 & 4,12 & 4,21 & 4,36 & 4,48 & 4,62 & 4,82 & 4,98 & 5,24 & 5,44 & 5,77 & 6,04 \\
\hline 0,35 & 3,61 & 3,72 & 3,80 & 3,94 & 4,04 & 4,17 & 4,35 & 4,49 & 4,72 & 4,91 & 5,21 & 5,45 \\
\hline 0,40 & 3,30 & 3,40 & 3,48 & 3,60 & 3,70 & 3,82 & 3,98 & 4,11 & 4,32 & 4,49 & 4,77 & 4,98 \\
\hline 0,45 & 3,05 & 3,14 & 3,22 & 3,33 & 3,42 & 3,53 & 3,68 & 3,80 & 4,00 & 4,15 & 4,41 & 4,61 \\
\hline 0,50 & 2,84 & 2,93 & 3,00 & 3,10 & 3,19 & 3,29 & 3,43 & 3,54 & 3,72 & 3,87 & 4,11 & 4,30 \\
\hline 0,75 & 2,17 & 2,24 & 2,29 & 2,37 & 2,43 & 2,51 & 2,62 & 2,70 & 2,84 & 2,95 & 3,14 & 3,28 \\
\hline 1,00 & 1,79 & 1,85 & 1,89 & 1,96 & 2,01 & 2,09 & 2,16 & 2,23 & 2,35 & 2,44 & 2,59 & 2,71 \\
\hline 1,25 & 1,54 & 1,59 & 1,63 & 1,68 & 1,73 & 1,78 & 1,86 & 1,92 & 2,02 & 2,10 & 2,23 & 2,33 \\
\hline 1,50 & 1,37 & 1,41 & 1,44 & 1,49 & 1,53 & 1,58 & 1,65 & 1,70 & 1,79 & 1,86 & 1,97 & 2,07 \\
\hline 1,75 & 1,23 & 1,27 & 1,30 & 1,35 & 1,38 & 1,43 & 1,49 & 1,54 & 1,62 & 1,68 & 1,78 & 1,86 \\
\hline 2,00 & 1,13 & 1,16 & 1,19 & 1,23 & 1,26 & 1,31 & 1,36 & 1,40 & 1,48 & 1,54 & 1,63 & 1,70 \\
\hline 2,25 & 1,04 & 1,06 & 1,10 & 1,14 & 1,17 & 1,21 & 1,26 & 1,30 & 1,37 & 1,42 & 1,51 & 1,58 \\
\hline 2,50 & 0,97 & 1,00 & 1,02 & 1,06 & 1,09 & 1,12 & 1,17 & 1,21 & 1,27 & 1,32 & 1,40 & 1,47 \\
\hline 3,00 & 0,85 & 0,89 & 0,91 & 0,94 & 0,96 & 1,00 & 1,04 & 1,07 & 1,13 & 1,17 & 1,24 & 1,30 \\
\hline 3,50 & 0,78 & 0,80 & 0,82 & 0,85 & 0,87 & 0,90 & 0,94 & 0,97 & 1,02 & 1,06 & 1,12 & 1,17 \\
\hline 4,00 & 0,71 & 0,73 & 0,75 & 0,78 & 0,80 & 0,82 & 0,86 & 0,88 & 0,93 & 0,97 & 1,03 & 1,07 \\
\hline 4,50 & 0,66 & 0,68 & 0,69 & 0,72 & 0,74 & 0,76 & 0,79 & 0,82 & 0,86 & 0,90 & 0,95 & 0,99 \\
\hline 5,00 & 0,61 & 0,63 & 0,65 & 0,67 & 0,69 & 0,71 & 0,74 & 0,76 & 0,80 & 0,83 & 0,88 & 0,93 \\
\hline 7,00 & 0,49 & 0,50 & 0,52 & 0,53 & 0,55 & 0,57 & 0,59 & 0,61 & 0,64 & 0,67 & 0,71 & 0,74 \\
\hline 10,0 & 0,39 & 0,40 & 0,41 & 0,42 & 0,43 & 0,45 & 0,47 & 0,48 & 0,51 & 0,53 & 0,56 & 0,58 \\
\hline 20,0 & 0,24 & 0,25 & 0,26 & 0,26 & 0,27 & 0,28 & 0,29 & 0,30 & 0,32 & 0,33 & 0,35 & 0,37 \\
\hline 30,0 & 0,18 & 0,19 & 0,20 & 0,20 & 0,21 & 0,21 & 0,22 & 0,23 & 0,24 & 0,25 & 0,27 & 0,28 \\
\hline
\end{tabular}


Tabela 5 - Concentrações de íons em água e respectivos riscos de toxicidade às plantas.

Table 5 - Water ion concentration and respective risks of toxication for the plants.

\begin{tabular}{|c|c|c|c|}
\hline \multirow[b]{2}{*}{ Íon } & \multicolumn{3}{|c|}{ Classes de toxicidade da água ${ }^{1}$} \\
\hline & $\begin{array}{c}\mathrm{T}_{1} \\
\text { Nenhum problema }\end{array}$ & $\begin{array}{c}\mathrm{T}_{2} \\
\text { Problema moderado }\end{array}$ & $\begin{array}{c}\mathrm{T}_{3} \\
\text { Problema severo }\end{array}$ \\
\hline Sódio ou Cloreto (mmol $\left.\mathrm{C}^{-1}:\right)$ & & & \\
\hline - Irrigação por superfície & $<3$ & $3-9$ & $>9$ \\
\hline - Irrigação por aspersão & $<3$ & $>3$ & \\
\hline Boro $\left(\mathrm{mg} \mathrm{L}^{-1}\right)$ & $<0,7$ & $0,7-3,0$ & $>3,0$ \\
\hline
\end{tabular}

Fonte: Adaptado de Ayers; Westcot (1991). ' Simbologia (T) não contida nos artigos originais; inserida neste item para resumir descrição da classe.

\section{CLASSIFICAÇÃ̃ DAS ÁGUAS PARA IRRIGAÇÃO NA REGIÃO DE BARAÚNA}

De conformidade com os resultados das análises químicas e os correspondentes limites de classificação, as classes de águas para a irrigação ficaram assim definidas (Tabela 6 e Figura 7): $70,8 \%$ das amostras analisadas se enquadram na classe $\mathrm{C}_{2} \mathrm{~S}_{1} \mathrm{~T}_{2}$, ou seja, são águas de média salinidade, baixa sodicidade e problema moderado de toxidez; $20,8 \%$ das amostras analisadas são do tipo $\mathrm{C}_{3} \mathrm{~S}_{1} \mathrm{~T}_{3}$ e neste caso são águas de alta salinidade, baixa sodicidade e problema severo de toxidez nas plantas; $8,4 \%$ são águas $\mathrm{C}_{2} \mathrm{~S}_{1} \mathrm{~T}_{1}$, ou seja, águas de média salinidade, baixa sodicidade e não apresentam nenhum problema de toxidez nas plantas. Na figura 7 apresenta a distribuição dos poços com a classificação das águas quanto ao uso para a irrigação.

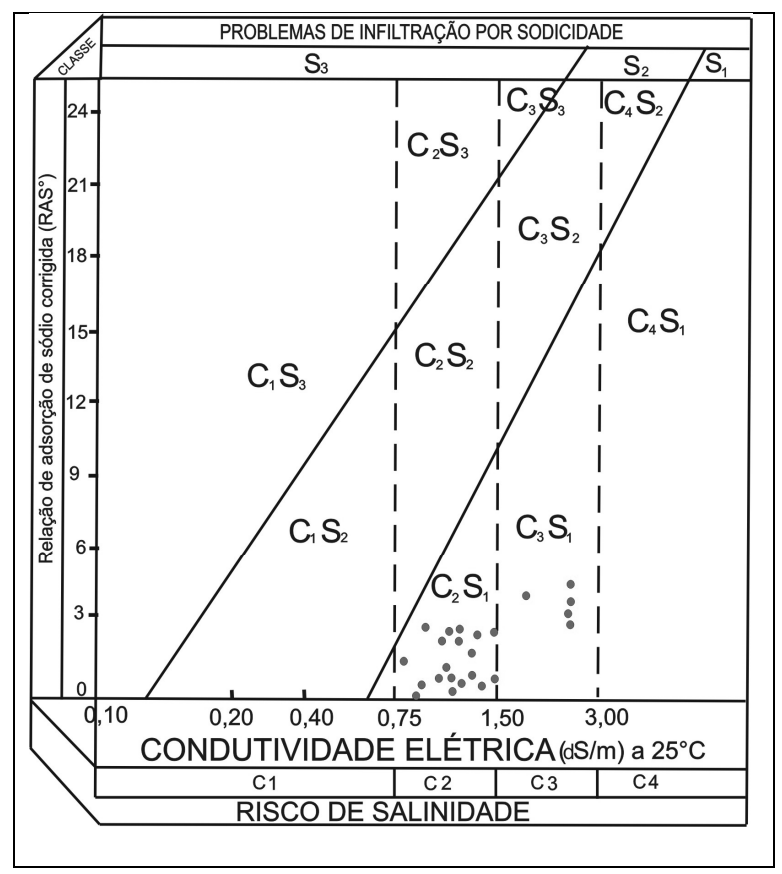

Figura 6 - Classificação das águas para irrigação

Figure 6 - Water classification for Irrigation

\section{CONCLUSÕES}

As águas subterrâneas do aqüífero Jandaíra, na região de Baraúna, estão sendo afetadas tanto do ponto de vista quantitativo como qualitativo, pelas atividades do desenvolvimento agrícola postas em prática na região.

Com efeito, foi verificada a ocorrência de um abaixamento acentuado da superfície potenciométrica do aquífero Jandaíra devido a explotação acentuada de poços que captam águas dos mesmos para o desenvolvimento da agricultura irrigada. Também, as águas em grande parte da área estão contaminadas por nitrato, com teores superiores a $10 \mathrm{mg} / \mathrm{L}$, que é o limite estabelecido para o uso humano (OMS).

Quanto ao uso das águas da região de Baraúna na irrigação, de um modo geral não há restrições, principalmente aquelas situadas nos setores oeste $\mathrm{e}$ noroeste da área que se enquadram nos tipos e $\mathrm{C}_{2} \mathrm{~S}_{1} \mathrm{~T}_{1}$ e $\mathrm{C}_{2} \mathrm{~S}_{1} \mathrm{~T}_{2}$. Algumas restrições são atribuídas às águas do tipo $\mathrm{C}_{3} \mathrm{~S}_{1} \mathrm{~T}_{3}$ que ocorrem na parte Central e Sul da área, haja vista que existem riscos de salinidade de solos e de rendimento das culturas.

As águas do setor sudeste da área são em geral do tipo $\mathrm{Ca}-\mathrm{Mg}-\mathrm{Cl}$, as quais estão associadas a presença de calcários com intercalações de folhelho e calcários relativamente puros; e no setores Norte e Oeste a maior facilidade de dissolução de calcários (nível de carste acentuado) resulta em águas do tipo $\mathrm{Ca}-\mathrm{HCO}_{3}$. 
Tabela 6 - Classes de água para irrigação

Table 6 - Water Classes for irrigation

\begin{tabular}{|c|c|c|c|c|c|c|c|c|c|}
\hline \multirow{2}{*}{ Poço } & $\mathrm{Na}^{+}$ & $\mathrm{Ca}^{2+}$ & $\mathrm{Mg}^{2+}$ & $\mathrm{Cl}^{-}$ & $\mathrm{HCO}_{3}^{-}$ & \multirow{2}{*}{$\begin{array}{c}\mathrm{CE} \\
(\mu \mathrm{S} / \mathrm{cm})\end{array}$} & \multirow{2}{*}{$\mathrm{pH}$} & \multirow{2}{*}{ RAS } & \multirow{2}{*}{ Classe } \\
\hline & \multicolumn{5}{|c|}{$(\mathrm{mmol} / \mathrm{L})$} & & & & \\
\hline $\mathrm{P}-05$ & 0,70 & 2,80 & 1,59 & 4,27 & 4,66 & 1,27 & 7,3 & 0,56 & $\mathrm{C}_{2} \mathrm{~S}_{1} \mathrm{~T}_{2}$ \\
\hline P-09 & 0,70 & 3,60 & 1,82 & 5,24 & 5,31 & 1,50 & 7,2 & 0,53 & $\mathrm{C}_{2} \mathrm{~S}_{1} \mathrm{~T}_{2}$ \\
\hline PB-017 & 1,81 & 1,87 & 1,12 & 2,86 & 3,42 & 0,84 & 7,4 & 1,62 & $\mathrm{C}_{2} \mathrm{~S}_{1} \mathrm{~T}_{1}$ \\
\hline PB-028 & 0,46 & 3,46 & 1,40 & 4,08 & 5,31 & 1,25 & 7,1 & 0,37 & $\mathrm{C}_{2} \mathrm{~S}_{1} \mathrm{~T}_{2}$ \\
\hline PB-052 & 0,46 & 3,04 & 1,21 & 3,11 & 5,53 & 1,06 & 7,2 & 0,41 & $\mathrm{C}_{2} \mathrm{~S}_{1} \mathrm{~T}_{2}$ \\
\hline PB-054 & 0,66 & 3,04 & 1,35 & 4,08 & 5,04 & 1,25 & 7,2 & 0,56 & $\mathrm{C}_{2} \mathrm{~S}_{1} \mathrm{~T}_{2}$ \\
\hline PB-069 & 2,36 & 2,66 & 1,40 & 4,24 & 4,32 & 1,09 & 7,3 & 1,84 & $\mathrm{C}_{2} \mathrm{~S}_{1} \mathrm{~T}_{2}$ \\
\hline PB-095 & 0,62 & 2,43 & 0,84 & 2,91 & 3,47 & 0,98 & 7,3 & 0,56 & $\mathrm{C}_{2} \mathrm{~S}_{1} \mathrm{~T}_{1}$ \\
\hline PB-118 & 6,21 & 4,49 & 2,29 & 11,61 & 5,75 & 1,92 & 7,2 & 4,28 & $\mathrm{C}_{3} \mathrm{~S}_{1} \mathrm{~T}_{3}$ \\
\hline PB-154 & 0,70 & 3,46 & 1,31 & 4,08 & 5,24 & 1,34 & 7,3 & 0,60 & $\mathrm{C}_{2} \mathrm{~S}_{1} \mathrm{~T}_{2}$ \\
\hline PB-161 & 0,66 & 2,90 & 1,35 & 3,88 & 4,76 & 1,27 & 7,4 & 0,55 & $\mathrm{C}_{2} \mathrm{~S}_{1} \mathrm{~T}_{2}$ \\
\hline PB-225 & 6,53 & 4,67 & 2,43 & 10,69 & 5,90 & 1,92 & 7,6 & 4,42 & $\mathrm{C}_{3} \mathrm{~S}_{1} \mathrm{~T}_{3}$ \\
\hline PB-229 & 6,92 & 3,75 & 1,87 & 9,68 & 5,38 & 1,92 & 7,8 & 5,22 & $\mathrm{C}_{3} \mathrm{~S}_{1} \mathrm{~T}_{3}$ \\
\hline PB-237 & 6,09 & 3,49 & 2,41 & 10,51 & 4,68 & 1,92 & 7,6 & 4,14 & $\mathrm{C}_{3} \mathrm{~S}_{1} \mathrm{~T}_{3}$ \\
\hline PB-247 & 7,15 & 3,74 & 2,85 & 13,64 & 3,20 & 2,06 & 7,6 & 4,29 & $\mathrm{C}_{3} \mathrm{~S}_{1} \mathrm{~T}_{3}$ \\
\hline PB-250 & 3,16 & 2,63 & 1,75 & 5,52 & 4,85 & 1,39 & 7,6 & 2,52 & $\mathrm{C}_{2} \mathrm{~S}_{1} \mathrm{~T}_{2}$ \\
\hline PB-251 & 2,77 & 3,15 & 1,50 & 4,75 & 5,79 & 1,32 & 7,5 & 2,30 & $\mathrm{C}_{2} \mathrm{~S}_{1} \mathrm{~T}_{2}$ \\
\hline PB-472 & 2,97 & 2,66 & 1,12 & 4,22 & 4,19 & 1,25 & 7,5 & 2,43 & $\mathrm{C}_{2} \mathrm{~S}_{1} \mathrm{~T}_{2}$ \\
\hline PB-502 & 3,81 & 2,62 & 2,52 & 8,66 & 3,34 & 1,50 & 7,6 & 2,57 & $\mathrm{C}_{2} \mathrm{~S}_{1} \mathrm{~T}_{2}$ \\
\hline PB-527 & 0,70 & 3,41 & 1,54 & 4,66 & 4,41 & 1,46 & 7,2 & 0,55 & $\mathrm{C}_{2} \mathrm{~S}_{1} \mathrm{~T}_{2}$ \\
\hline PB-534 & 2,33 & 3,12 & 1,27 & 3,96 & 5,19 & 1,20 & 7,7 & 2,00 & $\mathrm{C}_{2} \mathrm{~S}_{1} \mathrm{~T}_{2}$ \\
\hline PB-586 & 1,63 & 3,41 & 1,31 & 3,69 & 5,48 & 1,11 & 7,6 & 1,39 & $\mathrm{C}_{2} \mathrm{~S}_{1} \mathrm{~T}_{2}$ \\
\hline PB-594 & 0,46 & 2,57 & 1,21 & 3,30 & 4,03 & 1,06 & 7,2 & 0,39 & $\mathrm{C}_{2} \mathrm{~S}_{1} \mathrm{~T}_{2}$ \\
\hline PB-597 & 2,57 & 2,60 & 1,24 & 4,10 & 4,51 & 1,23 & 7,4 & 2,07 & $\mathrm{C}_{2} \mathrm{~S}_{1} \mathrm{~T}_{2}$ \\
\hline
\end{tabular}


Aspectos hidrogeoquímicos e classes de água do aqüífero cárstico Jandaíra para irrigação, Baraúna, RN

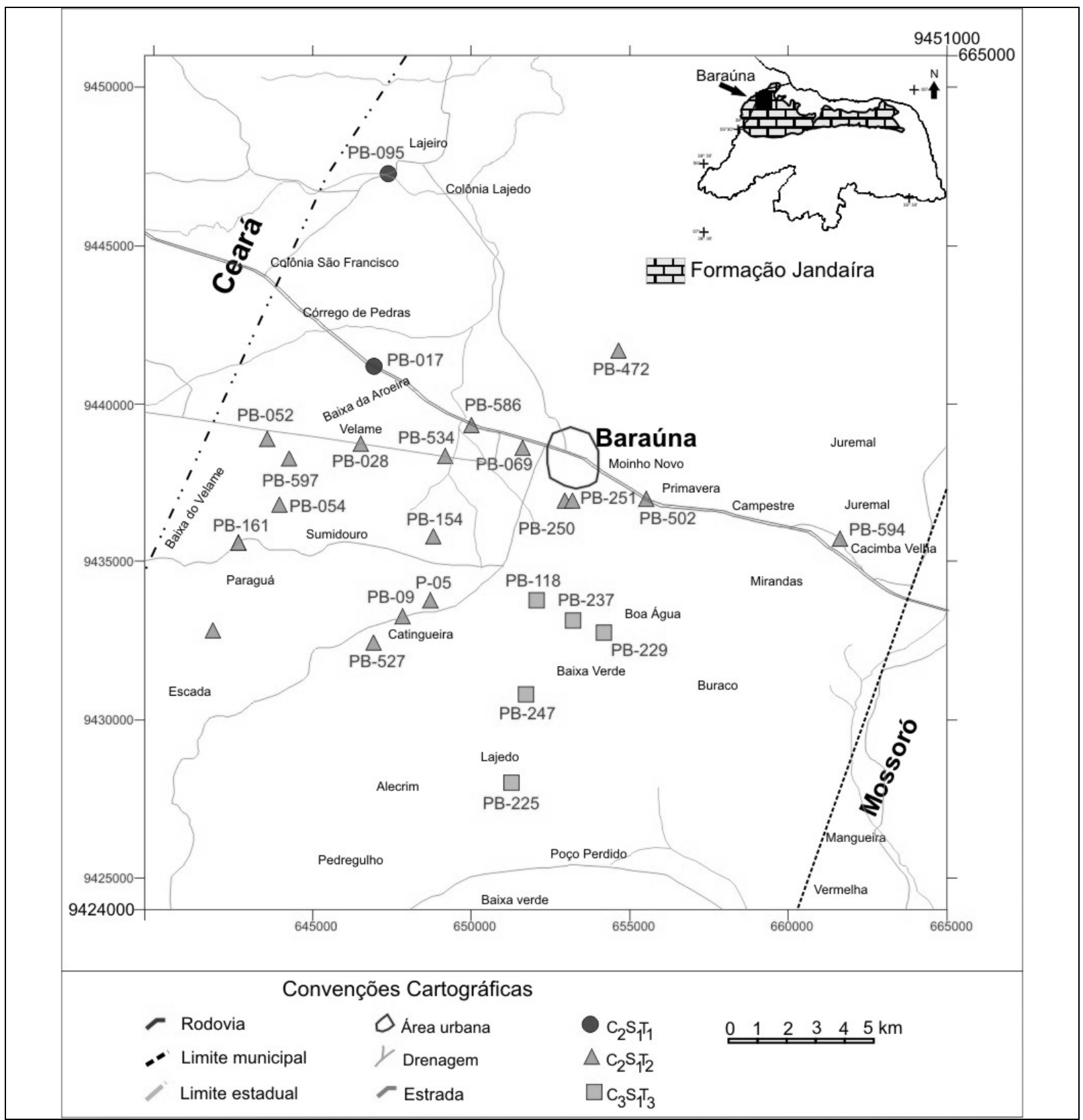

Figura 7 - Distribuição das classes de água quanto ao uso na irrigação.

Figure 7 - Water classes distribution for irrigation use. 


\section{REFERÊNCIAS}

AYERS, R.S., WESTCOT, D.W. A qualidade da água na agricultura. Trad. Gheyi, H. R., Medeiros, J. F., DAMASCENO, F.V. Campina Grande: UFPB. 218p. (Estudos FAO: Irrigação e Drenagem, 29 revisado1), 1991.

BHUMBLA, D.R. Chemical composition of irrigation water and its effect on crop growth and soil properties. In: Worthington, E. B. (ed.). Arid land irrigation in developing countries: Environmental problems and effects. Oxford: Pergamon Press, p.279-287, 1977.

BRASIL- Ministério da Saúde, Portaria n518 de 25 de março de 2004- Procedimentos e responsabilidades relativos ao controle e vigilância da qualidade da água para consumo humano e seu padrão de potabilidade, 2004.

DINIZ FILHO, W.D.O. FIGUEREDO, E.M. Diagnóstico preliminar das causas que afetam o rebaixamento de nível potenciométrico do aquífero Jandaíra - Região de Baraúna, RN. SERHID Secretaria de Recursos Hídricos do Rio Grande do Norte. (Relatório Técnico), 2003.

FEITOSA, E.C. O aquífero Jandaíra no município de Baraúna/ RN - Atualização dos conhecimentos. Secretaria de Recursos Hídricos do Rio Grande do Norte (SERHID) / Fundação de Apoio ao Desenvolvimento da UFPE (FADE), Natal, RN, 1994. FERNANDES, M.A.B, SANTIAGO, M.M.F., GOMES, D.F., Filho, J.M, FRICHKORN, H, LIMA, J.G.; A Origem dos Cloretos nas Águas Subterrâneas na Chapada do Apodi, Ceará. Revista Águas Subterrâneas, v.19, n 1, p.25-34, 2005.

FRENKEL, H. Reassessment of water quality criteria for irrigation. In: Shainberg. I, Shalhevet, J. Soil salinity under irrigation. Berlin: Springer-Verlag, p.143-72, 1984.

HOORN, J.W., ALPHEN, J.G. Salinity control; salt balance; and leaching requirement of irrigated soils. Wageningen: Agricultural University of Wageningen, 1981. (Lectures notes for the Twentyninth International Course on Land Drainage, 1990).
HOLANDA, J.S., AMORIM, J.R.A., 1992. Qualidade da água para irrigação. EMPARN/ EMBRAPA, Natal, RN.

MAAS, E.V.; Crop tolerance to saline sprinkling water. Plant and Soil, n.89, p.273-284, 1985.

MAAS, E.V. Salt tolerance of plants. Applied Agriculture Research, v.1, n.1,p.12-26, 1986.

MEDEIROS, A.B., MELO, J.G., VASCONCELOS, M.B., CASTRO, V.L. Aspectos hidrogeoquímicos e de qualidade das águas do aquífero Jandaíra na região de Baraúna, RN. XXI Simpósio de Geologia do Nordeste, Recife, Pe, 2005.

NUNES FILHO, J., GUERRA, C.A.M., SOUZA, A.R., SOARES, M.J.C.C. Qualidade da água de açudes para fins de irrigação no sertão de Pernambuco. Item - Irrigação e Tecnologia Moderna, Brasília, n.44, p.35-39, 1991.

PIZARRO, F. Drenaje agrícola y recuperacion de suelos salinos. 2.ed. Madrid: Editorial Española S.A., 1985.

RHOADES, J.D., KANDIAH, A., MASHAL, A.M. The use of saline water for crop production. Rome: FAO, 133p. (FAO:Irrigation and Drainage Paper, 48), 1992.

RICHARDS, L.A. Diagnosis and improvement of saline and alkali soils. Washington D.C.: U.S. Salinity Labratory. 160p. (USDA. Agriculture Handbook, 60), 1954.

SUAREZ, D.L. Relation between pHc and sodium adsorption ratio (SAR) and an alternative method of estimating SAR of soil or drainage waters. Soil Sci. Soc. Amer. J., n.45, p.469-75, 1981. 
